\title{
Effect of Erdosteine on COPD Exacerbations in COPD Patients with Moderate Airflow Limitation
}

This article was published in the following Dove Press journal:

International Journal of Chronic Obstructive Pulmonary Disease

\author{
Peter MA Calverley' \\ Clive Page $\mathbb{D}^{2}$ \\ Roberto W Dal Negro ${ }^{3}$ \\ Giovanni Fontana $\mathbb{I D}^{4}$ \\ Mario Cazzola (iD ${ }^{5}$ \\ Arrigo F Cicero ${ }^{6}$ \\ Edoardo Pozzi $\mathbb{1}^{7, \dagger}$ \\ Jadwiga A Wedzicha ${ }^{8}$ \\ 'Department of Medicine, Clinical \\ Sciences Centre, University Hospital \\ Aintree, Liverpool, UK; ${ }^{2}$ Faculty of Life \\ Sciences and Medicine, King's College, \\ London, UK; ${ }^{3}$ Lung Unit, National Centre \\ for Respiratory Pharmacoeconomics and \\ Pharmacoepidemiology, Verona, Italy; \\ ${ }^{4}$ Pulmonology Department, Cough \\ Centre, Careggi University Hospital, \\ Firenze, Italy; ${ }^{5}$ Department of Systems \\ Medicine, Chair of Respiratory Medicine, \\ University of Rome 'Tor Vergata', Rome, \\ Italy; ${ }^{6}$ Medical and Surgical Department, \\ University of Bologna, Bologna, Italy; \\ ${ }^{7}$ Medical Affairs Department, Edmond \\ Pharma, Paderno, Italy; ${ }^{8}$ Respiratory \\ Division, National Heart And Lung \\ Institute, Imperial College London, \\ London, UK
}

${ }^{\dagger}$ Dr Pozzii passed away on 9th July, 2019

Correspondence: Peter MA Calverley Department of Medicine, University Hospital Aintree, Lower Lane, Liverpool, Merseyside, UK

Tel +44 I5I 5295886

$\mathrm{Fax}+44$ I5I5295888

Email pmacal@liverpool.ac.uk
Background: The RESTORE study, a multi-national randomized, placebo-controlled study, showed that erdosteine - a muco-active antioxidant that modulates bacterial adhesiveness reduced the rate and duration of exacerbations in moderate and severe COPD with a history of exacerbations. How much benefit patients with less severe disease experience when taking this drug remains unclear.

Methods: This post hoc analysis of the 254 RESTORE participants with spirometricallydefined moderate COPD (post-bronchodilator forced expiratory volume in 1 second $\left[\mathrm{FEV}_{1}\right]$ $50-79 \%$ predicted) examined exacerbation rate and duration, time to first exacerbation, and exacerbation-free time. Data were analyzed using descriptive statistics and comparisons between treatment groups used Wilcoxon rank-sum tests, Mann-Whitney $U$-tests, or log rank tests.

Results: Patients with moderate COPD received erdosteine $300 \mathrm{mg}$ twice daily $(\mathrm{n}=126)$ or placebo $(\mathrm{n}=128)$ added to usual COPD therapy for 12 months. During this time, there were 53 exacerbations in the erdosteine group and 74 in the placebo group, with $42.1 \%$ and $57.8 \%$ of patients, respectively, experiencing an exacerbation. There was a $47 \%$ reduction in the mean exacerbation rate with erdosteine compared to placebo ( 0.27 vs 0.51 exacerbations per-patient per-year, respectively, $P=0.003$ ), and a $58.3 \%$ reduction in the mild exacerbation rate ( 0.23 vs 0.53 mild exacerbations perpatient per-year, $P=0.001)$. Mean duration of exacerbations was $26 \%$ shorter in erdosteine-treated patients ( 9.1 vs 12.3 days for placebo, $P=0.022$ ), with significant reductions in the duration of mild and moderate-to-severe exacerbations. Mean time to first exacerbation was prolonged by $7.7 \%$ (182 days for erdosteine vs 169 days for placebo, $P<0.001)$ and the mean exacerbation-free time was increased by 51 days ( 279 days for erdosteine vs 228 days for placebo; $P<0.001$ ).

Conclusion: These results indicate that adding erdosteine to usual COPD maintenance therapy reduces the number of mild, and duration of all, exacerbations in patients with moderate COPD and a history of exacerbations.

Keywords: antioxidant, anti-inflammatory, chronic obstructive pulmonary disease, erdosteine, COPD exacerbations

\section{Plain-Language Summary}

Patients with COPD often experience exacerbations, when their symptoms worsen and they require additional medication, emergency treatment, or hospital admission. A recent 1 year clinical trial found that COPD patients with moderate or severe breathing problems and a history of exacerbation events experienced fewer exacerbations if they took a medication called erdosteine, as well as their usual treatment for COPD. This study reexamined the clinical trial data by looking only at the group of patients with moderate breathing problems. The results showed that patients taking erdosteine had fewer exacerbations that not only took longer to occur, but also lasted a shorter amount of time. Thus, the overall burden of exacerbations was reduced in such patients. These findings suggest that erdosteine may be a useful medication for patients with less severe COPD. 


\section{Introduction}

Exacerbations of COPD are significant events for patients leading to a worse quality of life, use more health care resources, and are at an increased risk of dying. ${ }^{1}$ Preventing exacerbations is an important goal of COPD treatment, ${ }^{1,2}$ but exacerbation reduction remains suboptimal with current treatment strategies. ${ }^{3}$ As lung function declines more rapidly in the early stages of the disease, ${ }^{4,5}$ and is accelerated by exacerbations, ${ }^{6,7}$ increasing treatment intensity to prevent exacerbations in patients with moderately severe airflow obstruction might be a useful treatment strategy.

Exacerbations of COPD are generally classified as mild, moderate, or severe according to the treatment and/ or health care resource use required in their management. ${ }^{8}$ The duration of exacerbations can vary widely, but longer events are associated with greater morbidity, poorer health status, hospitalization, an increased risk of recurrence, and a faster decline in lung function. ${ }^{9-11}$ Time to first or subsequent exacerbation has been used as an outcome measure in some clinical trials. ${ }^{12,13}$ Moreover, determining exacerbation-free time is a useful way to assess the impact of exacerbations. ${ }^{14}$

A variety of treatment approaches to prevent exacerbations have been described, ${ }^{2}$ including medications that improve lung mechanics, ${ }^{13,15}$ or use of anti-inflammatory agents. ${ }^{16,17}$ The latter agents appear to be most effective in individuals with higher blood eosinophil counts, who are at increased risk of exacerbation. ${ }^{18,19}$ An alternative strategy is to use antioxidant-based therapies. However, until recently, the results of antioxidant therapy have largely been confined to patients living in Asia and at doses higher than recommended for safe use..$^{20,21}$

Erdosteine is an oral mucoactive agent with antioxidant and anti-inflammatory properties that also reduces bacterial adhesiveness and enhances the effects of antibiotic therapy; ${ }^{22}$ all of these properties may be useful for the prevention and treatment of COPD exacerbations. In the RESTORE (Reducing Exacerbations and Symptoms by Treatment with ORal Erdosteine in COPD) study, ${ }^{23} 467$ patients with spirometrically-defined moderate or severe COPD (stage II/III according to Global Initiative for Obstructive Lung Disease [GOLD] 2007 definition) were randomized to receive erdosteine (300 $\mathrm{mg}$ twice daily) or placebo for 12 months in addition to their usual COPD maintenance therapy. Erdosteine treatment was associated with a reduction in the rate and duration of exacerbations by $19.4 \%(P=0.01)$ and
$24.6 \%$ ( $P=0.023)$, respectively, compared with placebo, but there was no significant treatment effect on the time to first exacerbation. ${ }^{23}$ The reduced exacerbation rate was primarily due to a reduction in mild exacerbations. ${ }^{23}$

In the community setting, more than half of all COPD patients $(53.5 \%)$ have moderate COPD as defined using spirometry (post-bronchodilator forced expiratory volume in 1 second $\left[\mathrm{FEV}_{1}\right] 50-79 \%$ predicted). ${ }^{24,25}$ In this post hoc analysis of the RESTORE study, we aimed to determine the effectiveness of erdosteine on COPD exacerbations in the subgroup of patients who had spirometrically-defined moderate COPD.

\section{Methods}

\section{Study Design And Patients}

The RESTORE study (NCT01032304) was a Phase III multinational, randomized, double-blind, placebo-controlled study conducted in 10 European countries. Full details of the study design, inclusion/exclusion criteria, ethical approval and results have been reported. ${ }^{23}$ Briefly, following a 2-week runin period of continued usual COPD therapy, 467 patients with moderate or severe COPD (grade II/III, GOLD 2007 classification) were randomized to receive either oral erdosteine (300 mg twice daily, $\mathrm{n}=228$ ) or placebo $(\mathrm{n}=239)$ for 12 months in addition to their usual COPD therapy. Written informed consent was provided by each participant prior to enrolment. The trial was conducted in accordance with the Declaration of Helsinki and ethical approval was granted locally as outlined in the Supplementary appendix.

For this post hoc analysis, we reclassified patients participating in the RESTORE study using the spirometry criteria from the GOLD 2017 guidelines. ${ }^{1}$ Thus, patients with moderate COPD (GOLD 2) were defined as having a postbronchodilator $\mathrm{FEV}_{1}$ between $50 \%$ and $79 \%$ predicted, and patients with severe COPD (GOLD 3) had a postbronchodilator $\mathrm{FEV}_{1}$ between $30 \%$ and $49 \%$ predicted; both subgroups had a post-bronchodilator fixed ratio $\mathrm{FEV}_{1} /$ forced vital capacity $[\mathrm{FVC}]<0.70$. Participants were outpatients aged 40-80 years, current or ex-smokers ( $\geq 10$ pack-years), on a stable therapeutic regimen for $\geq 8$ weeks prior to inclusion who had experienced $\geq 2$ acute COPD exacerbations requiring medical intervention in the previous 12 months, but with no exacerbations in the preceding 2 months.

\section{Outcome Measures}

A COPD exacerbation was defined as a worsening of symptoms beyond normal day-to-day variation that required 
a change in regular medication and/or health care resource utilization. ${ }^{26}$ Exacerbations were confirmed by the investigators from the variation in daily symptom (dyspnea, cough, sputum) scores, changes in regular medication, use of additional medication or emergency hospitalization for COPD, as recorded in the patient diary. The severity of each exacerbation was graded by investigators as mild, moderate or severe (Table 1). The duration of an exacerbation was determined from the diary card data. The onset of a COPD exacerbation was defined by the presence of at least two days of symptomatic worsening and/or an increased level of health care utilization. Resolution of a mild exacerbation was determined by a return to pre-event symptomatology. Similarly, the end of a moderate or severe exacerbation was determined as the cessation of additional treatment and return to background therapy or hospital discharge and return to background therapy. At least 10 consecutive days without symptom variation and/or additional medication and/or re-hospitalization since the previous episode were required before a new event could be registered. Exacerbation-free time was defined as the number of days without an exacerbation present while the patient was participating in the study. All diary card assessments were conducted blind to treatment allocation as described in the appendix to the primary publication ${ }^{23}$ and explained in the supplement to this manuscript.

Other outcomes measured during the RESTORE study, but not used in this post hoc analysis, include physician assessment of disease severity, the St George's Respiratory Questionnaire, hospitalization rate, morning pre-dose $\mathrm{FEV}_{1}$, FVC, use of reliever medication, and the 6-min walk test; these have been reported in detail by Dal Negro et al. ${ }^{23}$

Safety assessments included monitoring adverse events. Blood samples were collected at baseline (randomization) and at the end of the study (after 12 months of treatment) for evaluation of clinical laboratory parameters, including blood eosinophil counts.

\section{Statistical Analysis}

All post hoc efficacy analyses were conducted using intention-to-treat (ITT) principles on randomized patients who received at least one dose of study treatment and had at least one available post-baseline efficacy evaluation. Likelihood-based methods were used to handle missing data. The adverse events reported for the moderate COPD subgroup were based on all patients randomized to treatment.

Baseline characteristics are reported using descriptive statistics (means and standard deviations [SD] or percentages). Comparisons between treatment groups were performed using the chi-squared test followed by Fisher's exact test.

The frequency of exacerbations was analyzed using a Poisson mixed regression model, with correction for overdispersion and including the following covariates: treatment, age, sex, body mass index, and $\mathrm{FEV}_{1}$ at baseline, as described previously. ${ }^{23,27}$ The COPD exacerbation rate over the 12-month follow-up period was calculated as the mean exacerbation rate per-patient per-year. Differences in exacerbation rates between treatment groups were analyzed nonparametrically using the Wilcoxon rank-sum test (data are reported as odds rations [OR] with $95 \%$ confidence intervals $[\mathrm{CI}])$.

The duration of an exacerbation was calculated as the sum of days the patient was affected by a COPD exacerbation, as defined earlier. Time to first exacerbation was calculated as the time in days from the beginning of the study to the first exacerbation. Time free from exacerbation was calculated as the difference between the total days the patient was involved in the study and the number of days during which the patient experienced a COPD exacerbation. Comparisons between treatment groups used the MannWhitney $U$-test for exacerbation duration and the log rank test for time to first exacerbation.

All exacerbation analyses were repeated by concomitant use/non-use of inhaled corticosteroids (ICS).

Table I Classification Of Exacerbation Severity In The RESTORE Study Based On Health Care Utilization ${ }^{26}$

\begin{tabular}{|l|l|}
\hline $\begin{array}{l}\text { Exacerbation } \\
\text { Severity }\end{array}$ & Level Of Health Care Utilization \\
\hline Mild & Patient has an increased need for medication (bronchodilators), which he/she can manage in own normal environment \\
\hline Moderate & $\begin{array}{l}\text { Patient has increased need for medication (antibiotics and/or systemic corticosteroids) and feels the need to seek } \\
\text { additional medical assistance }\end{array}$ \\
\hline Severe & $\begin{array}{l}\text { Patient/caregiver recognizes obvious and/or rapid deterioration in condition, requiring hospitalization or an emergency } \\
\text { department visit }\end{array}$ \\
\hline
\end{tabular}


Blood eosinophil counts are summarized using descriptive statistics. Exacerbation rate among patients with moderate COPD was analyzed by stratification of baseline blood eosinophil counts $(<150$ cells $/ \mu \mathrm{L}$ and $\geq 150$ cells/ $\mu \mathrm{L}$ ) in keeping with previous reports in patients not using dual bronchodilator therapy. ${ }^{19}$

Statistical analyses were performed using SPSS version 21.0 (IBM, Armonk, NY, USA). A two-sided $P$-value $<0.05$ was considered nominally significant for all tests in this post hoc analysis.

\section{Results}

Of the 407 patients randomized, 254 had a postbronchodilator $\mathrm{FEV}_{1}>50 \%$ predicted. Of these patients with moderate COPD, 126 received erdosteine and 128 placebo, as detailed in the modified CONSORT figure (Figure 1). The baseline characteristics of the subgroups of patients in this post-hoc analysis are summarized in Table 2 together with those of the total RESTORE population. ${ }^{23}$ The patients receiving either erdosteine or placebo were similar in this subgroup analysis but, as expected, the $\mathrm{FEV}_{1}$ and $\mathrm{FVC}$ values were significantly higher in the subgroup with moderate COPD than in the subgroup with severe COPD, while ICS were used less frequently in moderate COPD (Table 2).

There were 127 exacerbations during the 12 months of treatment in the moderate COPD subgroup $(\mathrm{n}=254): 53$ in the erdosteine group ( $42.1 \%$ of patients) and 74 in the placebo group (57.8\% of patients).

In the subgroup of patients with moderate COPD, there was a $47 \%$ reduction in the mean exacerbation rate with erdosteine treatment compared to placebo $(0.27$ vs 0.51 exacerbations per-patient per-year; $P=0.003$; Figure 2): OR: $0.53 ; 95 \%$ CI: $0.32-0.87 ; P=0.017$. This compares to the $19.4 \%$ reduction seen for the total RESTORE population (Figure 2). In contrast, there were no differences in the exacerbation rate between active and placebo arms in the patients with an $\mathrm{FEV}_{1}<50 \%$ predicted. In the subgroup with moderate COPD, erdosteine treatment was associated with a $58.3 \%$ reduction in the rate of mild exacerbations ( 0.23 vs 0.53 mild exacerbations perpatient per-year in the erdosteine and placebo groups, respectively; OR: $0.41 ; 95 \%$ CI: $0.37-0.54 ; P=0.001$ ). For the total RESTORE population, the reduction in the rate of mild exacerbations was $57.1 \%$ ( 0.23 vs 0.54 mild exacerbations per-patient per-year in the erdosteine and placebo groups, respectively; $P=0.002){ }^{23}$
The mean duration of exacerbations was $26 \%$ shorter in patients with moderate COPD treated with erdosteine: 9.1 (SD 7.4) days compared with 12.3 (SD 9.6) days for the placebo-treated patients $(P=0.022)$ (Table 3$)$. The difference between treatments was significant for the duration of mild exacerbations and of moderate-to-severe exacerbations in this subgroup (Table 3). However, in the subgroup with severe COPD, the duration of exacerbations was similar for the erdosteine and placebo-treated patients (Table 3). In addition, there was no difference in exacerbation frequency and duration between moderate COPD patients who were ICS users and non-users $(P>0.05)$ (Supplementary Tables 1 and 2).

For patients with moderate COPD, the time to first exacerbation was $7.7 \%$ longer in the erdosteine group: mean 182 (SD 19) days vs 169 (SD 25) days in the placebo group; difference between treatment groups 13 days (95\% CI: 8.93-17.07), $P<0.001$. The effect of erdosteine on the time to first exacerbation in this subgroup was not influenced by the concomitant use of ICS (Supplementary Table 3).

Mean exacerbation-free time during the study period was significantly longer by a mean of 51 days $(P<0.001)$ in the erdosteine group compared with the placebo group for the patients with moderate COPD, and by a mean of 39 days $(P=0.008)$ for all RESTORE patients (Figure 3 ).

In both subgroups of patients with moderate COPD and severe COPD, there were no significant differences in blood eosinophil counts between treatment groups or changes over time within each treatment group, but patients with severe COPD had significantly higher eosinophil counts than those with moderate COPD at both time points and in both treatment groups (Supplementary Table 4). Among patients with moderate COPD, the mean exacerbation rate was higher in patients with blood eosinophil counts $\geq 150$ cells $/ \mu \mathrm{L}$, but erdosteine treatment significantly reduced the mean exacerbation rate compared with placebo in both subgroups with $<150$ cells $/ \mu \mathrm{L}$ and $\geq 150$ cells $/ \mu \mathrm{L}$ (Figure 4 ).

A total of 305 adverse events were reported during the study in the subgroup of patients with moderate COPD: 136 adverse events in the erdosteine group (3 serious adverse events) and 169 in the placebo group (4 serious adverse events). None of the serious adverse events was considered treatment-related or resulted in patient death. Erdosteine appeared to be well tolerated with no substantial differences from placebo. 


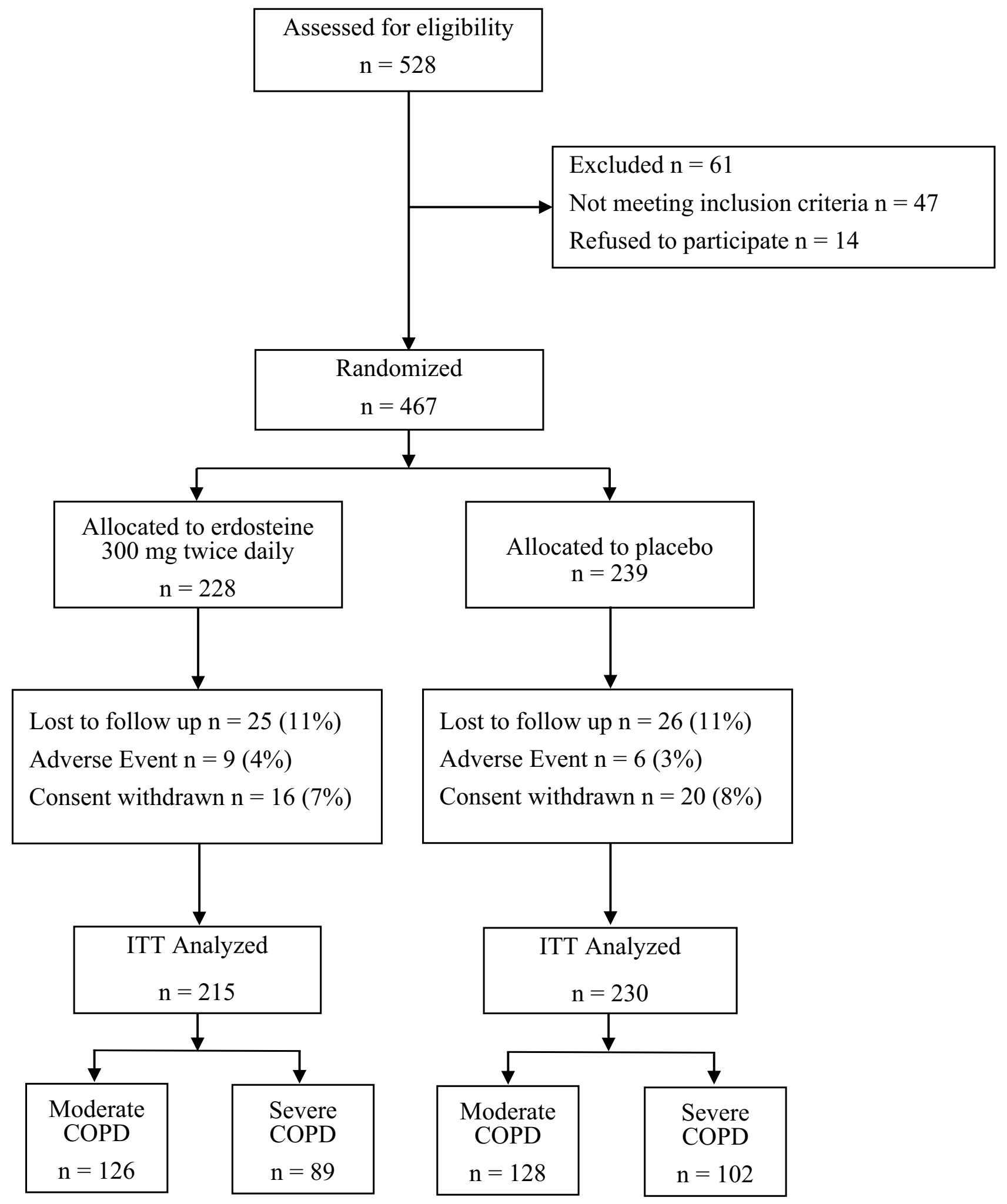

Figure I Patient disposition in RESTORE study.

\section{Discussion}

The RESTORE study demonstrated that erdosteine administered twice daily at the licensed dose $(300 \mathrm{mg})$ reduced the overall exacerbation rate in COPD patients with a history of exacerbations in the previous year, many of whom were already receiving previously standard therapy with $\mathrm{ICS}^{23}$ 
Table 2 Demographic And Baseline Characteristics Of Patients (ITT population)

\begin{tabular}{|c|c|c|c|c|c|c|}
\hline & \multicolumn{2}{|c|}{ Moderate COPD ${ }^{a}(\mathrm{~N}=254)$} & \multicolumn{2}{|c|}{ Severe COPD $(N=\mid 91)$} & \multicolumn{2}{|c|}{ All RESTORE Patients ${ }^{c}(\mathrm{~N}=445)$} \\
\hline & Erdosteine & Placebo & Erdosteine & Placebo & Erdosteine & Placebo \\
\hline Patients, $\mathrm{n}$ & 126 & 128 & 89 & 102 & 215 & 230 \\
\hline Age, years & $64.8(7.6)$ & $66.1(7.3)$ & $62.9(8.9)$ & $63.1(8.8)$ & $63.8(8.3)$ & $64.1(8.2)$ \\
\hline Male, \% & 65.9 & 72.7 & 74.5 & 73.9 & 71.8 & 74.6 \\
\hline BMI, $\mathrm{kg} / \mathrm{m}^{2}$ & $27.6(5.0)$ & $28.2(5.6)$ & $27.0(4.8)$ & $27.8(5.1)$ & $27.2(5.3)$ & $28.0(5.4)$ \\
\hline \multicolumn{7}{|l|}{ Smoking status, \% } \\
\hline Current smoker & 31.7 & 28.9 & 25.8 & 27.5 & 27.1 & 28.0 \\
\hline Ex-smoker & 68.3 & 71.1 & 74.2 & 72.5 & 72.9 & 72.0 \\
\hline ICS, ${ }^{\mathrm{d}} \mathrm{n}(\%)$ & $88(69.8)$ & 91 (7I.I) & $80(89.9)^{*}$ & $94(92.2)^{*}$ & $165(75.8)$ & $173(75.2)$ \\
\hline $\mathrm{FEV}_{1}, \mathrm{~L}$ & $1.6 \mathrm{I}(0.35)$ & $1.68(0.42)$ & $1.26(0.39)$ & $1.23(0.43)$ & $\mathrm{I} .43(0.40)$ & $\mathrm{I} .46(0.47)$ \\
\hline $\mathrm{FEV}_{1}, \%$ predicted & $59.88(6.3)$ & $61.08(6.8)$ & $47.21(10.83)$ & 46.72 (II.69) & $51.45(12.82)$ & $54.38(13.33)$ \\
\hline FVC, L & $2.82(0.66)$ & $2.89(0.72)$ & $2.59(0.99)^{*}$ & $2.54(0.97)^{*}$ & $2.74(0.93)$ & $2.74(0.94)$ \\
\hline Post-BD FEV $/$ /FVC, ratio $\%$ & $58.76(8.8)$ & $58.00(8.1)$ & $51.92(9.88)^{*}$ & $50.81(10.03)^{*}$ & $54.01(11.3)$ & $53.26(10.8)$ \\
\hline
\end{tabular}

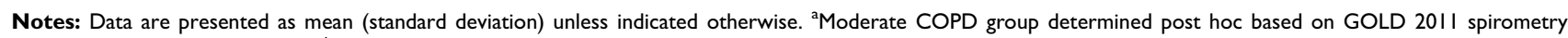
criteria (FEVI 50-79\% predicted); ' Severe COPD group determined post hoc based on GOLD 201 I spirometry criteria (FEVI $30 \%-49 \%$ predicted); ${ }^{\circ}$ Data from Dal Negro et $\mathrm{al} ;{ }^{23 \mathrm{~d}} \mathrm{ICS}$ alone or combined with adrenergic agents. ${ }^{*} \mathrm{P}<0.05$ versus moderate COPD group. (Categorical variables have been compared as continuous depending on the distribution: if normal with $t$-tested for independent samples, if not normal with comparisons between treatment groups used the Mann-Whitney $U$-test). All comparisons between the erdosteine and placebo groups were non-significant.

Abbreviations: BD, bronchodilator; BMI, body mass index; FEVI, forced expiratory volume in I second; FVC, forced vital capacity; ICS, inhaled corticosteroid; ITT, intention-to-treat; NS, not significant for comparison between treatment groups.

The primary report of the RESTORE study did not seek to identify which subgroup of patients would benefit the most from erdosteine treatment, so we addressed this issue in the current exploratory post hoc analysis. The results suggest that erdosteine use has a significant effect on exacerbations in patients with less severe COPD defined spirometrically $\left(\mathrm{FEV}_{1} 50-79 \%\right)$, as the relative effect of treatment on the overall exacerbation rate was almost doubled compared to placebo. A similar effect was seen on mild exacerbations classified by treatment intensity, but there was no reduction in the number of exacerbations observed in patients with severe COPD. Similarly, the reduction in the duration of mild exacerbations was most evident in patients with moderate COPD. In this subgroup, erdosteine therapy was associated with a significant increase in both the time to first exacerbation and the total time without exacerbations in patients with a history of exacerbations. Unlike other antiinflammatory agents, the positive effects of erdosteine were not restricted to patients with a higher blood eosinophil count. Encouragingly, there was no evidence of a significant adverse event profile in these less impaired COPD patients. These findings potentially impact the way in which we view COPD exacerbations and for the treatment options to be considered for preventing exacerbations.

Stratification of the treatment effect by baseline lung function has been examined with other treatments known to prevent COPD exacerbations, including inhaled bronchodilators ${ }^{15}$ and long-acting inhaled bronchodilator-ICS combinations. ${ }^{28}$ In general, categorizing patients in this way has little impact on the overall efficacy of these treatments, although the results of the FLAME (Effect of Indacaterol Glycopyronium vs Fluticasone Salmeterol on COPD Exacerbations) study indicated that inhaled long-acting bronchodilator combination therapy may be relatively less effective in patients with very severe COPD ( $\mathrm{FEV}_{1}<30 \%$ predicted, GOLD stage IV). ${ }^{13}$ However, applying this approach to the RESTORE study data gave a different answer. Although the ITT analysis reported a $19 \%$ reduction in exacerbation rate of the total study population, this difference was driven by patients in the spirometrically-defined moderate COPD subgroup, where a $47 \%$ reduction in exacerbation rate was seen with erdosteine therapy compared to placebo. By contrast, there was little difference in the exacerbation rate 


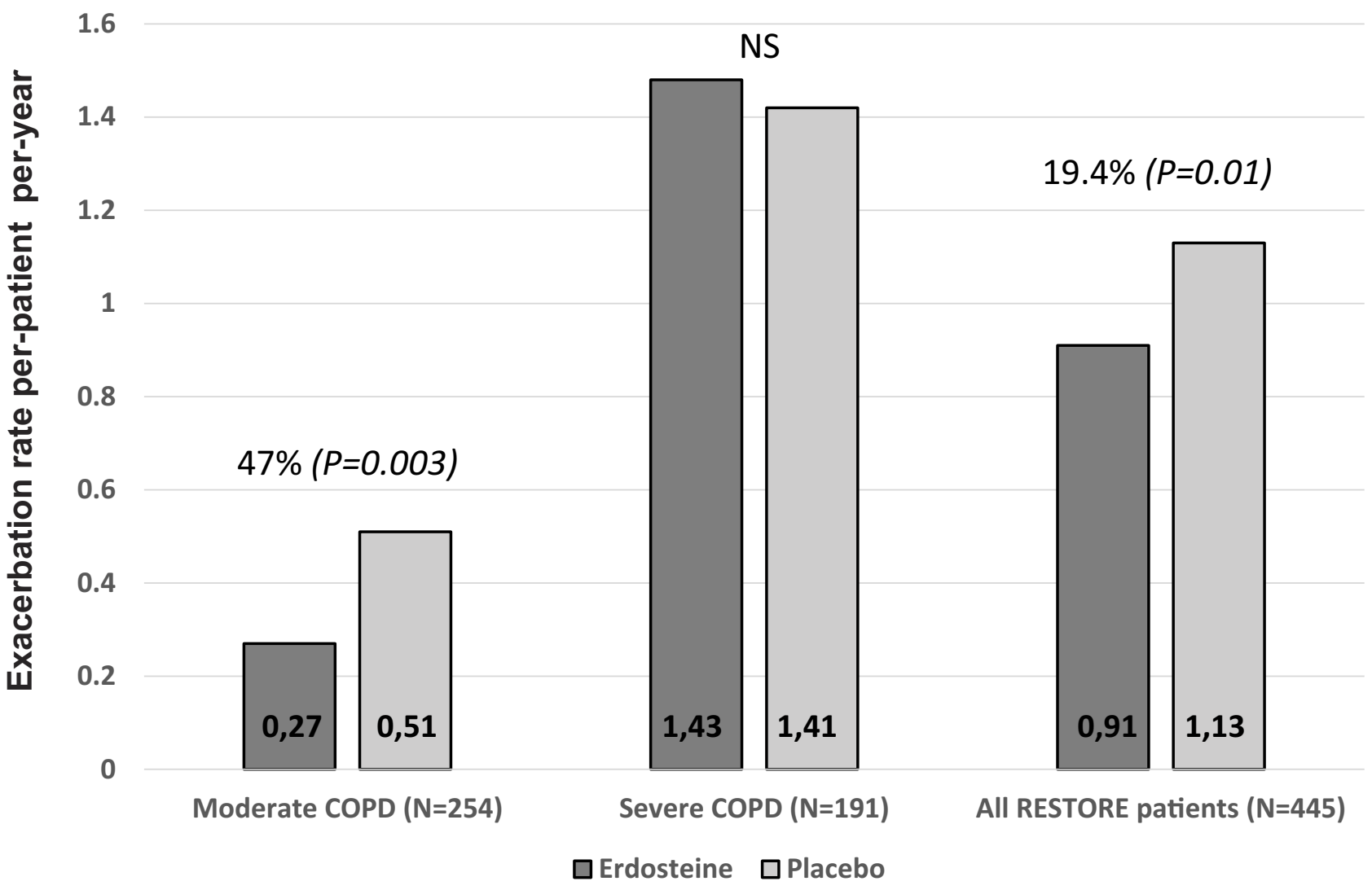

Figure 2 Exacerbation rate according to COPD severity subgroup and for all patients in the RESTORE study.

among patients with worse spirometry when erdosteine therapy was compared with placebo. Although there were fewer patients in this severe COPD subgroup, it seems unlikely that the comparison is underpowered statistically given the lack of difference in event rates between the two treatment groups.
Recent reports have identified differences in the way that exacerbations are treated dependent on the background therapy taken. ${ }^{29}$ The SUMMIT (Study to Understand Mortality and Morbidity) study included patients with spirometricallydefined moderate COPD as in the RESTORE study but, unfortunately, did not characterize exacerbations as carefully

Table 3 Exacerbation Duration (In days) By Exacerbation Severity (Mild Or Moderate-To-severe), COPD Severity And Treatment Group

\begin{tabular}{|l|l|l|l|l|}
\hline & Erdosteine & Placebo & \%Change & P-value \\
\hline Moderate COPD patients (N=254) & & & \\
All exacerbations & $9.1(7.4)$ & $12.3(9.6)$ & $-26.0(5.6)$ & 0.022 \\
Mild exacerbations & $7.7(4.9)$ & $9.8(8.0)$ & $-21.4(5.0)$ & $-23.4(4.8)$ \\
Moderate-to-severe exacerbations & $10.5(8.5)$ & $13.7(10.4)$ & & 0.037 \\
\hline Severe COPD patients (N=191) & & & $-14.8(4.7)$ \\
All exacerbations & $10.9(7.5)$ & $12.8(9.9)$ & $-16.3(4.8)$ & $-7.3(3.4)$ \\
Mild exacerbations & $8.7(5.8)$ & $10.4(7.8)$ & & 0.093 \\
Moderate-to-severe exacerbations & $13.9(9.8)$ & & 0.131 \\
\hline All RESTORE patients (N=445) & & $12.6(9.7)$ & $-24.6(5.3)$ \\
All exacerbations & $9.5(7.2)$ & $10.4(8.2)$ & $-19.2(4.9)$ & $-21.3(5.2)$ \\
Mild exacerbations & $8.4(5.2)$ & $14.1(10.8)$ & 0.023 \\
Moderate-to-severe exacerbations & $11.1(8.9)$ & 0.039 \\
\hline
\end{tabular}

Note: Data are presented as mean (standard deviation) in days. 


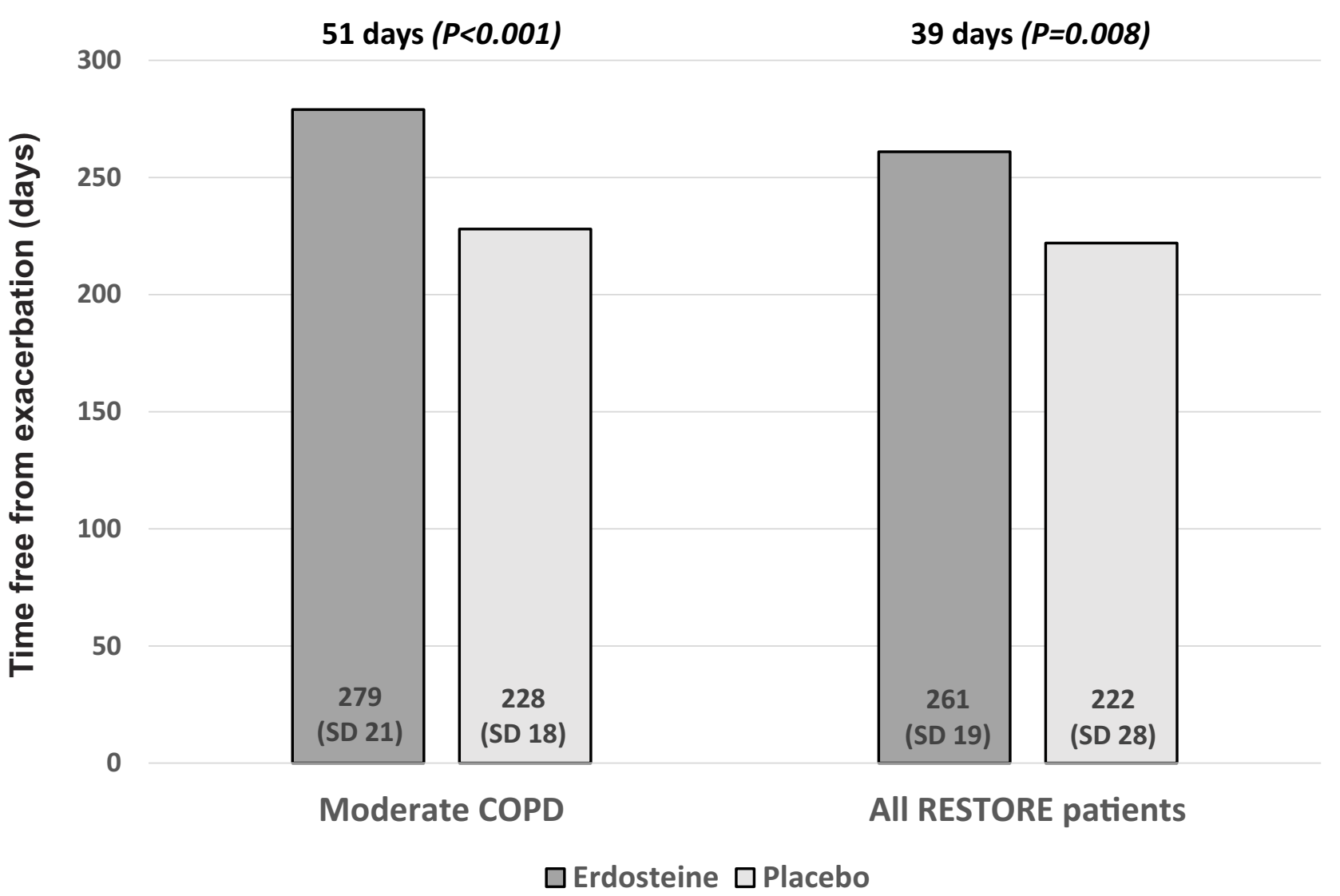

Figure 3 Exacerbation-free time (mean days) in patients with moderate COPD and for all patients in the RESTORE study.

as we did and so data about mild events, where routine treatment is intensified but new therapy is not started, were not available in that large study. ${ }^{29}$ In the present analysis, we found significant reductions in the duration of mild exacerbations in the subgroup of patients with moderate COPD treated with erdosteine and this played an important role in increasing the time to first event for all exacerbations. Although meta-analysis of a number of studies of mucolytic drugs suggest that they are useful in preventing COPD exacerbations, ${ }^{30}$ such an effect has not been reported in individual studies, which likely reflects their differing study designs and inclusion criteria. Our findings of a longer time to first exacerbation and a longer exacerbation-free time with erdosteine in patients with spirometrically-defined moderate COPD lends support to the major effect of this drug being in patients with milder airflow obstruction.

A particular strength of the RESTORE study was the prespecified recording of exacerbation duration. To date, only regular macrolide therapy has been shown to decrease exacerbation duration, ${ }^{31}$ which is a known risk factor for subsequent hospitalization in COPD patients. ${ }^{14}$ As anticipated, exacerbations did not last as long in patients with moderate COPD as compared to severe disease, and exacerbations classified as mild were shorter than those where antibiotics and/or corticosteroids were used. However, in all these subgroups, erdosteine treatment was associated with shorter exacerbation events compared with placebo, a difference that was statistically significant in patients with moderate spirometric impairment. Given the greater variation in exacerbation duration in the subgroup with severe COPD, it is likely that comparisons in these patients are underpowered to detect a significant difference; although, if present, the difference between erdosteine and placebo groups is likely to be of smaller magnitude than in the patients with moderate COPD. Overall the total number of days that patients experienced symptoms related to an exacerbation was lower in the erdosteine treated subjects when compared to those receiving placebo in addition to their usual treatments. This may be relevant as data from population studies and early disease cohorts suggests that exacerbations including unreported events have an important impact on patient health status and well-being at an earlier stage of COPD than previously appreciated. ${ }^{32,33}$ 


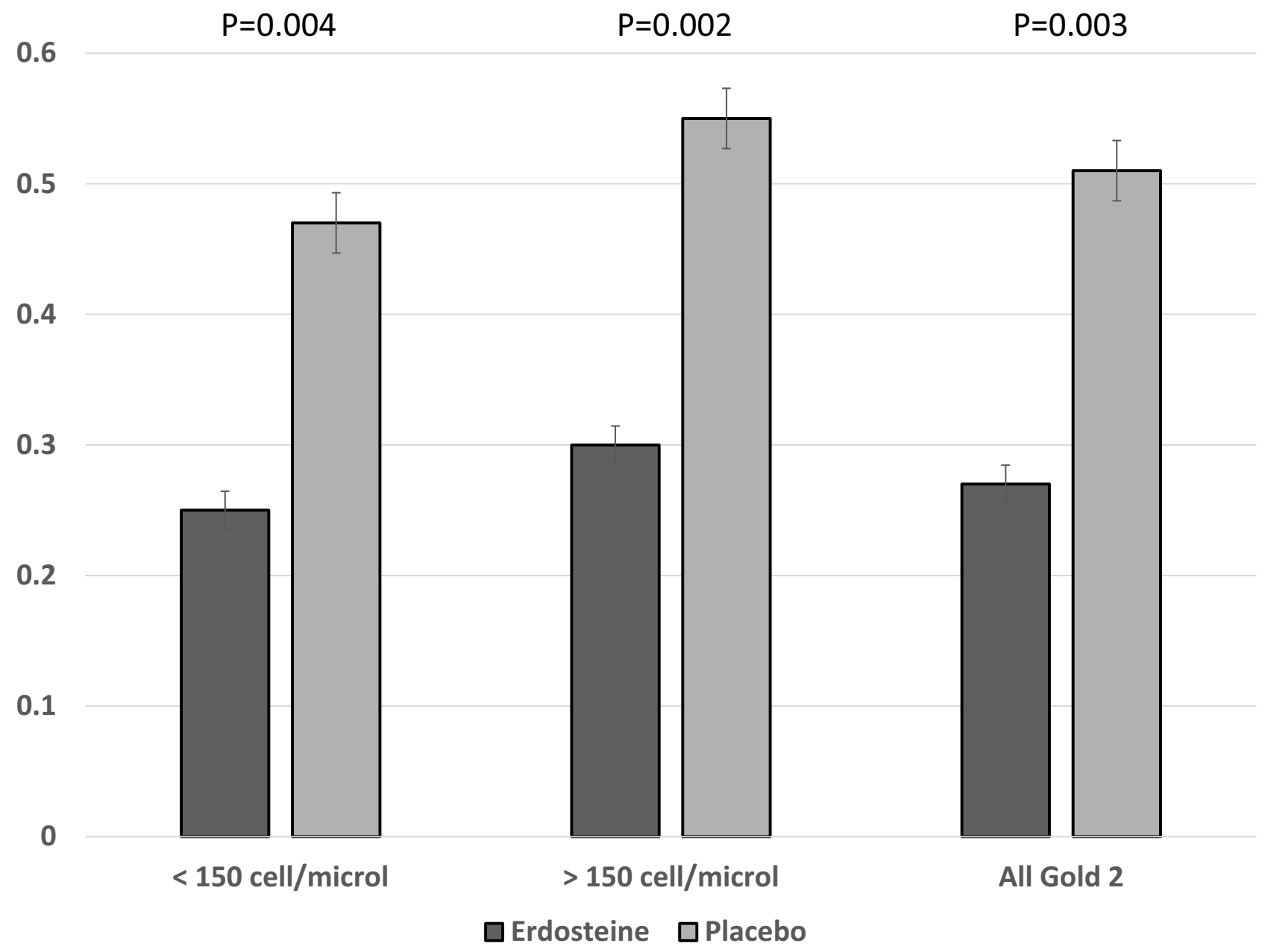

Figure 4 Exacerbation rate by blood eosinophil count $(<150$ cells $/ \mu \mathrm{L}$ and $\geq 150$ cells $/ \mu \mathrm{L})$ for patients with moderate COPD by treatment group.

There has been much discussion about the use of the blood eosinophil count as a stratifying variable when selecting anti-inflammatory therapy for patients with COPD. Like others we found that patients with higher blood eosinophil counts experienced more exacerbations, but unlike data obtained from patients treated with ICS and roflumilast ${ }^{18,19}$ we saw no differential effect of treatment based on eosinophil numbers. However, our event rates were relatively low and we could not stratify for higher eosinophil counts as was done in some other trials. Nonetheless, the similarity of the mean data in the high and low eosinophil groups supports the idea that erdosteine's effects are not substantially influenced by the blood eosinophil count.

Our study has several strengths beyond data collection of mild exacerbation events. Erdosteine was well tolerated in both subgroups of patients with moderate and severe COPD. This likely reflects its use at licensed doses rather than the high doses of other antioxidants, such as $\mathrm{N}$-acetylcysteine (NAC), which were needed to show an effect on exacerbation rate in patients with COPD. ${ }^{21}$ Concerns about the potential of further harm at high-dose NAC has led some investigators to curtail clinical research with this formulation. ${ }^{34}$ As erdosteine had so few side effects there was no substantial difference in the withdrawal rate from either treatment arm (see Figure 1), a factor that has complicated interpretation of other studies. ${ }^{7,35}$ Our patients were predominantly Caucasian and were using appropriate background medication throughout the study, including ICS in $75 \%$ of patients. An earlier European trial found no benefit of NAC in patients with COPD who used ICS. ${ }^{36}$ In the present analysis, we found no interaction between ICS use and the effects of therapy in our patients with moderate COPD in terms of exacerbation number or duration. Nonetheless, there are limitations to our analysis. This is a pre-specified post hoc analysis and so the significance levels we report should be considered as only 
nominally significant. We did not have information that would allow us to classify patients according to the current GOLD system, so our patients must be considered to be in either groups $\mathrm{C}$ or $\mathrm{D}$ of that classification system. ${ }^{1}$ The entry criteria for the RESTORE study specified that patients should have a prior exacerbation history, but the mean exacerbation rate in the placebo arm of the study was lower than expected. This is not unique to our study, having been seen in other treatment studies with similar recruitment criteria. ${ }^{37,38}$ This lower than expected exacerbation rate in the RESTORE study may reflect an improved patient adherence to existing therapy on study entry or the marked variability in exacerbations over time as seen in recent reports from the large SPIROMICS (SubPopulations and InteRmediate Outcome Measures In COPD Study) and TIOSPIR (TIOtropium Safety and Performance in Respimat $($ ) studies, where a large percentage of participants with a history of frequent exacerbations did not subsequently experience these events during 2-3 years of follow-up in the study. ${ }^{39,40}$ The use of diary cards to define events can be criticized as being imprecise, but it has been used widely by other groups ${ }^{6,9-11}$ and the methodology used here has been covered in detail in the appendix to the original RESTORE publication ${ }^{23}$. Given the blinded nature of the study we believe the data on number and duration of events are likely to be correct.

This post hoc analysis has a number of clinical implications. It provides further evidence for the heterogeneity of exacerbation events, at least in terms of their response to therapy with differences in the ability of an oral antioxidant like erdosteine to prevent events according to the severity of the patient's airflow obstruction and the type of treatment thought necessary to manage the episode. Whether this reflects differences in the etiology of the exacerbations, the host response to the triggering factor, or a combination of these two remains unclear. However, the present results do emphasize the value of knowing the severity of airflow obstruction when considering what prophylactic therapy to prescribe as erdosteine did not reduce the number of moderate or severe events reported by our patients. Currently, there is renewed interest in the factors in early COPD leading to disease progression, ${ }^{41}$ and a need to identify well tolerated treatments that can be used earlier in the natural history of COPD than those previously considered. Our data suggests that drugs like erdosteine, which are orally active, well tolerated and can prevent exacerbation events that are common in less severe disease, may prove useful in the management of the less severely impaired COPD patient with a history of exacerbations.

\section{Acknowledgements}

Funding, medications and investigator's meeting costs for this study were provided by Edmond Pharma. Funding information for this article has been deposited with the Crossref Funder Registry. Medical writing assistance was provided by Deirdre Elmhirst of Elmhirst Scientific Consultancy Limited, funded by Edmond Pharma. The authors do not plan to share further data beyond that included in this paper. We wish to acknowledge the substantial contribution of our late colleague Dr Edoardo Pozzi who died soon after this paper was submitted. His drive and commitment were crucial to ensuring that this project was completed. This paper was presented at the European Respiratory Society 2018 as a poster presentation with interim findings. The poster's abstract was published in "Poster Abstracts" in the European Respiratory Journal https://erj.ersjournals.com/content/52/suppl_62/PA776

\section{Author Contributions}

All authors contributed to data analysis, drafting or revising the article, gave final approval of the version to be published, and agree to be accountable for all aspects of the work.

\section{Disclosure}

EP is employed by Edmond Pharma. PC, CP, RDN, GF, $\mathrm{MC}$, and AC report personal fees from Edmond Pharma. The authors report no other conflicts of interest in this work.

\section{References}

1. Vogelmeier CF, Criner GJ, Martinez FJ, et al. Global strategy for the diagnosis, management, and prevention of chronic obstructive lung disease 2017 report: GOLD executive summary. Am J Respir Crit Care Med. 2017;195:557-582. doi:10.1164/rccm.201701-0218PP

2. Wedzicha JA, Calverley PMA, Albert RK, et al. Prevention of COPD exacerbations: a European Respiratory Society/American Thoracic Society guideline. Eur Respir J. 2017;50(3):1602265. doi:10.1183/ 13993003.02265-2016

3. Miravitlles M, D’Urzo A, Singh D, Koblizek V. Pharmacological strategies to reduce exacerbation risk in COPD: a narrative review. Respir Res. 2016;17(1):112. doi:10.1186/s12931-016-0425-5

4. Tantucci C, Modina D. Lung function decline in COPD. Int J COPD. 2012;7:95-99. doi:10.2147/COPD

5. Drummond MB, Hansel NN, Connett JE, Scanlon PD, Tashkin DP, Wise RA. Spirometric predictors of lung function decline and mortality in early chronic obstructive pulmonary disease. Am J Respir Crit Care Med. 2012;185(12):1301-1306. doi:10.1164/rccm.201202$0223 \mathrm{OC}$ 
6. Donaldson GC, Seemungal TA, Bhowmik A, Wedzicha JA. Relationship between exacerbation frequency and lung function decline in chronic obstructive pulmonary disease. Thorax. 2002;57:847-852. doi:10.1136/thorax.57.10.847

7. Vestbo J, Edwards LD, Scanlon PD, ECLIPSE Investigators, et al. Changes in forced expiratory volume in 1 second over time in COPD. N Engl J Med. 365;2011:1184-1192. doi:10.1056/NEJMoa1105482

8. Oliveira AS, Munhá J, Bugalho A, et al; GI DPOC Grupo de Interesse na Doenca Pulmonar Obstrutiva Cronica. Identification and assessment of COPD exacerbations. Pulmonology. 2018;24:42-47.

9. Aaron SD, Donaldson GC, Whitmore GA, Hurst JR, Ramsay T, Wedzicha JA. Time course and pattern of COPD exacerbation onset. Thorax. 2012;67:238-243. doi:10.1136/thoraxjnl-2011-200768

10. Perera WR, Hurst JR, Wilkinson TM, et al. Inflammatory changes, recovery and recurrence at COPD exacerbation. Eur Respir J. 2007;29:527-534. doi:10.1183/09031936.00092506

11. Donaldson GC, Law M, Kowlessar B, et al. Impact of prolonged exacerbation recovery in chronic obstructive pulmonary disease. Am J Respir Crit Care Med. 2015;192:943-950. doi:10.1164/rccm.201412-2269OC

12. Vogelmeier C, Hederer B, Glaab T, et al; POET-COPD Investigators. Tiotropium versus salmeterol for the prevention of exacerbations of COPD. $N$ Engl J Med. 2011;364(12):1093-1103. doi:10.1056/ NEJMoa1008378

13. Wedzicha JA, Banerji D, Chapman KR, et al. Indacaterolglycopyrronium versus salmeterol-fluticasone for COPD. $N \mathrm{Engl}$ $J$ Med. 2016;374:2222-2234. doi:10.1056/NEJMoa1516385

14. Boer LM, Bischoff EW, Borgijink X, et al. 'Exacerbation-free time' to assess the impact of exacerbations in patients with chronic obstructive pulmonary disease (COPD): a prospective observational study. NPJ Prim Care Respir Med. 2018;28(1):12. doi:10.1038/s41533-018-0079-5

15. Calverley PMA, Anzueto AR, Carter K, et al. Tiotropium and olodaterol in the prevention of chronic obstructive pulmonary disease exacerbations (DYNAGITO): a double-blind, randomised, parallel-group, active-controlled trial. Lancet Respir Med. 2018;6 (5):337-344. doi:10.1016/S2213-2600(18)30102-4

16. Lipson DA, Barnhart F, Brealey N, et al. Once-daily single-inhaler triple versus dual therapy in patients with COPD. $N$ Engl J Med. 2018;378(18):1671-1680. doi:10.1056/NEJMoa1713901

17. Wedzicha JA, Calverley PM, Rabe KF. Roflumilast: a review of its use in the treatment of COPD. Int J Chron Obstruct Pulmon Dis. 2016;11:81-90.

18. Martinez FJ, Rabe KF, Calverley PMA, et al. Determinants of response to roflumilast in severe COPD: pooled analysis of two randomized trials. Am J Respir Crit Care Med. 2018;198(10):12681278. doi:10.1164/rccm.201712-2493OC

19. Bafadhel M, Peterson S, De Blas MA, et al. Predictors of exacerbation risk and response to budesonide in patients with chronic obstructive pulmonary disease: a post-hoc analysis of three randomised trials. Lancet Respir Med. 2018;6(2):117-126. doi:10.1016/S22132600(18)30006-7

20. Zheng JP, Kang J, Huang SG, et al. Effect of carbocisteine on acute exacerbation of chronic obstructive pulmonary disease (PEACE Study): a randomised placebo-controlled study. Lancet. 2008;371:2013-2018. doi:10.1016/S0140-6736(08)60869-7

21. Zheng JP, Wen FQ, Bai CX, et al; PANTHEON study group. Twice daily $\mathrm{N}$-acetylcysteine $600 \mathrm{mg}$ for exacerbations of chronic obstructive pulmonary disease (PANTHEON): a randomised, double-blind placebo-controlled trial. Lancet Respir Med. 2014;2:187-194. doi:10.1016/S2213-2600(13)70286-8

22. Moretti M. Pharmacology and clinical efficacy of erdosteine in chronic obstructive pulmonary disease. Exp Rev Respir Med. 2007;1:307-316.

23. Dal Negro RW, Wedzicha JA, Iversen M; RESTORE group, et al. Effect of erdosteine on the rate and duration of COPD exacerbations: the RESTORE study. Eur Respir J. 2017;50:1700711. doi:10.1183/ 13993003.00711-2017
24. Vestbo J, Vogelmeier C, Small M, Higgins V. Understanding the GOLD 2011 strategy as applied to a real-world COPD population. Respir Med. 2014;108:729-736. doi:10.1016/j.rmed.2014.03.002

25. Raluy-Callado M, Lambrelli D, MacLachlan S, Khalid JM. Epidemiology, severity, and treatment of chronic obstructive pulmonary disease in the United Kingdom by GOLD 2013. Int J Chron Obstruct Pulmon Dis. 2015;10:925-937.

26. Rodriguez-Roisin R. Toward a consensus definition for COPD exacerbations. Chest. 2000;117:398-401. doi:10.1378/chest.117.5_ suppl_2.398S

27. Keene ON, Calverley PM, Jones PW, Vestbo J, Anderson JA. Statistical analysis of exacerbation rates in COPD: TRISTAN and ISOLDE revisited. Eur Respir J. 2008;32:17-24.

28. Jenkins CR, Jones PW, Calverley PM, et al. Efficacy of salmeterol/ fluticasone propionate by GOLD stage of chronic obstructive pulmonary disease: analysis from the randomised, placebo-controlled TORCH study. Respir Res. 2009;10:59. doi:10.1186/1465-9921-10-59

29. Martinez FJ, Vestbo J, Anderson JA, et al. Effect of fluticasone furoate and vilanterol on exacerbations of chronic obstructive pulmonary disease in patients with moderate airflow obstruction. Am J Respir Crit Care Med. 2017;195(7):881-888. doi:10.1164/ rccm.201607-14210C

30. Cazzola M, Rogliani P, Calzetta L, Hanania NA, Matera MG. Impact of mucolytic agents on COPD exacerbations: a pair-wise and network meta-analysis. COPD. 2017;14:552-563. doi:10.1080/15412555. 2017.1347918

31. Seemungal TA, Wilkinson TM, Hurst JR, Perera WR, Sapsford RJ, Wedzicha JA. Long-term erythromycin therapy is associated with decreased chronic obstructive pulmonary disease exacerbations. Am J Respir Crit Care Med. 2008;178(11):1139-1147. doi:10.1164/ rccm.200801-1450C

32. Woodruff PG, Barr RG, Bleecker E, et al. Clinical significance of symptoms in smokers with preserved pulmonary function. $N$ Engl J Med. 2016;374(19):1811-1821. doi:10.1056/NEJMoa1505971

33. Labonté LE, Tan WC, Li PZ, et al; Canadian Respiratory Research Network; CanCOLD Collaborative Research Group. Undiagnosed chronic obstructive pulmonary disease contributes to the burden of health care use. Data from the CanCOLD Study. Am J Respir Crit Care Med. 2016;194(3):285-298. doi:10.1164/rccm.201509-1795OC

34. Johnson K, McEvoy CE, Naqvi S, et al. High-dose oral $\mathrm{N}$-acetylcysteine fails to improve respiratory health status in patients with chronic obstructive pulmonary disease and chronic bronchitis: a randomized, placebo-controlled trial. Int J Chron Obstruct Pulmon Dis. 2016;11:799-807.

35. Calverley PM, Spencer S, Willits L, Burge PS, Jones PW, Group IS. Withdrawal from treatment as an outcome in the ISOLDE study of COPD. Chest. 2003;124(4):1350-1356. doi:10.1378/chest.124.4. 1350

36. Decramer M, Rutten-van Mölken M, Dekhuijzen PN, et al. Effects of $\mathrm{N}$-acetylcysteine on outcomes in chronic obstructive pulmonary disease (Bronchitis Randomized on NAC Cost-Utility Study, BRONCUS): a randomised placebo-controlled trial. Lancet. 2005;365(9470):1552-1560. doi:10.1016/S0140-6736(05)66456-2

37. Calverley PM, Kuna P, Monso E, et al. Beclomethasone/formoterol in the management of COPD: a randomised controlled trial. Respir Med. 2010;104(12):1858-1868. doi:10.1016/j.rmed. 2010.09.008

38. Papi A, Vestbo J, Fabbri L, et al. Extrafine inhaled triple therapy versus dual bronchodilator therapy in chronic obstructive pulmonary disease (TRIBUTE): a double-blind, parallel group, randomised controlled trial. Lancet. 2018;391(10125):1076-1084. doi:10.1016/ S0140-6736(18)30206-X

39. Han MK, Quibrera PM, Carretta EE, SPIROMICS investigators, et al. Frequency of exacerbations in patients with chronic obstructive pulmonary disease: an analysis of the SPIROMICS cohort. Lancet Respir Med. 5;2017:619-626. doi:10.1016/S2213-2600(17)30207-2 
40. Calverley PM, Tetzlaff K, Dusser D, et al. Determinants of exacerbation risk in patients with COPD in the TIOSPIR study. Int $J$ Chron Obstruct Pulmon Dis. 2017;12:3391-3405. doi: $10.2147 /$ COPD
41. Martinez FJ, Han MK, Allinson JP, et al. At the root: defining and halting progression of early chronic obstructive pulmonary disease. Am J Respir Crit Care Med. 2018;197(12):1540-1551. doi:10.1164/ rccm.201710-2028PP

\section{Publish your work in this journal}

The International Journal of COPD is an international, peer-reviewed journal of therapeutics and pharmacology focusing on concise rapid reporting of clinical studies and reviews in COPD. Special focus is given to the pathophysiological processes underlying the disease, intervention programs, patient focused education, and self management protocols. This journal is indexed on PubMed Central, MedLine and CAS. The manuscript management system is completely online and includes a very quick and fair peer-review system, which is all easy to use. Visit http://www.dovepress.com/testimonials.php to read real quotes from published authors. 